\title{
Learners' reflexivity and the development of an e-learning community among students in China
}

Zhen $\mathrm{Li}^{*}$

School of Education, University of Southampton, Southampton, UK

(Received 15 August 2010; final version received 5 December 2010)

\begin{abstract}
The experiences of Chinese learners on two e-learning programmes in China were investigated, focusing particularly on the formation of learning communities. Data were collected using a range of instruments to access the learners' perspectives in depth and detail. Archer's account of reflexivity as the mediating power between structure and agency is applied to understanding how learners succeeded in one programme in forming a learning community, through their negotiated responses to the existing structural and cultural conditions, whereas little evidence was found of the emergence of learning community in the other case. Further understanding emerges from reappraisal of Confucian philosophy of learning and social relationships, how these influenced the participants' prior learning experiences and how they play a part in their responses to the e-learning experience.
\end{abstract}

Keywords: learning community; reflexivity; Archer; Chinese learners; e-learning

\section{Introduction}

The focus of learning theorisation has moved in recent years from individual behaviour and psychology to social interaction and practice, recognising in doing so the significant role of context in the learning process. The unit of analysis has moved from learners as bounded biological organisms, independent of context, to socially located "communities of practice" (Lave and Wenger 1991) or "activity systems" (Cole and Engeström 1993).

In studies of e-learning a similar shift in focus has widely occurred, from seeing technology as an individualised instructional tool to portraying it as a more discursive and communicative medium (Conole, Smith, and White 2007). Attention has been drawn to pedagogical innovations such as computer-supported collaborative learning that reflect the contemporary dominance of socio-constructivist learning approaches. e-Learning design has increasingly focused on the capacity of digital networks to support collaborative knowledge-building and a learning environment to facilitate learners' collaboration, online communication and reflection, to promote the formation of a learning community. Conole (2007) argues, however, that many of these innovations remain as ideas only and may not have appeared as actual changes in practice.

Despite the fact that the term "community of practice" (Wenger 1998) has been widely used and discussed in e-learning design and practice, the nature and develop-

*Email: zl@soton.ac.uk 
ment of learning communities through the actual experiences of e-learners, from their own perspectives, are still under-explored. Some initiatives have been taken in the United Kingdom to understand learners' use and experience of technology across programmes and subject disciplines (for example, Creanor et al. 2006; Conole et al. 2006) but more attention is still needed from the perspective of learners' experiences if we are aiming to facilitate the emergence of learning communities in e-learning. It should be kept in mind that it is their - the learners' - communities that we hope will emerge and it is therefore important to examine their experiences from their own perspectives. This paper uses two e-learning programmes in China as case studies to explore this issue; in each of these, a range of methods was used to collect data on the learners' e-learning experiences.

\section{Theoretical framework}

This study rejects technological determinism in e-learning research and analyses the actual experiences of learners in developing a learning community. In doing so, it draws on Margaret Archer's $(2000,2007)$ work on reflexivity as the mediating power between structure and agency, defined as "the regular exercise of the mental ability ... [of people] ... to consider themselves in relation to their (social) context and vice versa" (Archer 2007, 4). The pursuit of any social practice entails interplay between the causal powers of subjects themselves (agency) and those of relevant structural and cultural properties (structure). Through agential reflexivity, these two sets of causal power interact and an active role is thus restored to the individual agent. In this section, key elements of Archer's analytical framework will be outlined, with a more detailed engagement appearing in the later discussion of the study's findings.

Archer distinguishes between the existence of structural and cultural properties and subjects' responses to them. Herein lies the important part played by reflexivity: people can "design and determine their responses to the structured circumstances in which they found themselves, in the light of what they personally care about most" (Archer 2007, 11). The causal power of structural and social properties has to be activated by agents through social action. Archer $(2007,13)$ argues that the "deliberative process involved ... is emotionally charged rather than being a simple exercise in instrumental rationality, because ... our emotions (as distinct from moods) are commentaries on our concerns". By employing Archer's theoretical framework in e-learning contexts and recognising reflexivity as a distinct human power, this research treats learners as active rather than passive agents, who can exercise some governance in their own lives and learning

If we are to understand the interactions between the learners and the e-learning programmes in this study as forms of socio-cultural action, then, following Archer (1996, xxi), we must acknowledge that any such action takes place in a "context of innumerable interrelated theories, beliefs and ideas which had developed prior to it, and ... exert a conditional influence on it". The cultural context within which the learners in the study are working and to which their previous learning experience belongs is that of contemporary China. In order to understand better this context and these experiences, the study reappraises the nature and processes of learning in the philosophical tradition of Confucianism, in recognition of its tremendous influence on education in China. It challenges over-simplified (and often erroneous) interpretations of Confucian writings on learning and their miss-application in 
practice. In the Confucian tradition education is understood as a whole person constructing and transforming his/her life experience. Practice lies at the heart of this process and Confucian learning is a process of constantly practicing 'Ren' (仁, benevolence). This significance of action gives the Confucian educational tradition a close connection with western thinkers, such as Archer, who claim the centrality of practice in the emergence and development of our sense of self and all forms of knowledge.

\section{The study}

Two e-learning programmes were chosen as case studies: Case One is a Sino-UK collaborative MA e-learning programmed signed for in-service teachers; Case Two is a part-time BA e-learning programme for adult learners, delivered through a Chinese university. In Case One, 87 in-service teachers based at six different schools participated in the programme and each of them was assigned to study one of the three modules in the programme. In Case Two, 250 students from a range of locations across the country responded to an initial survey and became the subjects of the study. Each of these students was studying one of five different subjects in the programme. The cases were chosen on two grounds: there is a striking difference in the course design origins - one mainly due to western practitioners inspired by social constructivist approaches, the other based on a traditional didactic model; and they target similar populations. Learners' responses to the different designs are therefore highlighted through the comparison; at the same time the similarity in the learners' social and cultural backgrounds allows a degree of generalisability, which is often problematic in case studies.

Data were collected through five main tools: questionnaires, individual and focus group interviews, digital audio learning diaries, informal discussions and tutor reports. Quantitative data gathered by questionnaires provided key characteristics of the learners and their backgrounds, and identified appropriate individuals to participate in the qualitative phases of the research. In this paper, the evidence is mainly drawn from the detailed accounts of learners' experiences obtained from the instruments other than questionnaires: there are four focus groups interviews with 87 learners conducted in Case One, and two focus groups plus 22 individual interviews with 35 learners together in Case Two, combined with tutor reports, informal discussions with course designers and administrators on the sites in both cases.

In addition to holding interviews, a students' digital audio diary approach was used in Case Two to capture learners' experiences and interactions with greater immediacy and in their natural settings. For adult learners with a full-time job commitment, keeping a written diary is time-consuming (Timms et al. 2004), while an audio diary allowed the capture of day-to-day events that were significant to the learners without imposing a serious burden.

Data from the various qualitative instruments were organised and coded according to emerging patterns. Archer's account of reflexivity and emergence of social identity enabled the difference between the 'existence' of cultural and structural properties and the learners' 'responses' to them to emerge from the data, revealing the process of learners' reflexively interacting with the e-learning environment. There were striking differences in learners' experiences in responding to the different learning models in these two cases. 


\section{Findings and discussions}

The constructivist design in Case One was inspired by the desire to build a shared community of practice, with learners learning collaboratively. All the materials were transferred to an online format and were trialled in a virtual learning environment. There was a mix of resources and activities in every section of each study module: case studies; reading and reflective writing (via a journal); forum discussions; and three assignments. Learners were expected to finish all the learning materials within a certain time, but the form of assessment was not made clear from the outset, since it was not considered to be a necessary part of the piloting process. Far from building a shared community, however, learning was passive and chaotic. Most learners did not fully appreciate the instructional design in the way that the designers had expected. For instance, there was generally a lack of understanding of the purpose and use of the online discussion forum that was a part of the programme design.

Sometimes I logged in to the course website, but didn't know what to do, so I logged out again. Since we really haven't got much spare time after the whole day's work, it is impossible to spend so much time to find out what to do instead of actually doing it. (Case One: Student Focus Group 1)

I tried to post my question or respond to others in the forum, but it's very frustrating because I'm not sure how it works. Does it only allow commenting on a thread once or you have to comment at least a couple of times? (Case One: Student Focus Group 2)

Consequently, neither sufficient in-depth discussion nor effective collaboration developed. Most learners were seeking synchronous communication during their study but the chatting facility in the virtual learning environment was reported to be "poorly functioning" since it was not valued in the programme design. A learning community failed to emerge in this case.

In contrast, the learning model in Case Two was more content-driven and didactic. The learning objectives, course demand and assessment procedures were very explicit and very similar to the conventional programmes delivered on campus. Learning materials were designed for individual use. Online discussion forums were mainly designed for Teaching Assistants to answer learners' questions and solve technical problems. No collaborative learning activity was promoted by the course designers and tutors. Interestingly here, in compensating for the shortage of teacher-student interaction in the formal e-learning settings in the course, a high level of self-help and spontaneous collaboration emerged, both online and offline, amongst the learners. Learners made intensive use of the communicative tools they used in everyday life as well as the forum provided by the school to build up an intensive network amongst themselves. They organised learning groups to exchange information, solve problems, tackle assignments and prepare for examinations. Learners from the same city also organised social events for people to meet with each other from time to time. Learners' interviews and diary entries illuminate this phenomenon:

The best thing about coming to this course is that I got to know so many people who are doing the same course. We are varied in age, profession and location. But we help each other and learn from each other. Many of us became very good friends. This is something really wonderful (Case Two: Student Interview 1). 
Learning depends on good understanding, understanding depends on good communication. Sometimes participating in communication seems more important than giving the right answers (Case Two: Student Diary Entries 5:2)

Learning communities emerged in this case despite a lack of intention in the course design. Learners created their own social networks to support their learning, tailored to their particular needs rather than being constrained within those provided by the courses. They had strong personal views on how technologies may or may not support their learning activities.

Archer's theorisation of reflexivity and social identity can help us to understand the contrasting findings in these two cases and the very nature of the emergence of learning community among students.

\section{A brief summary of Archer's (2000) theorising of social identity}

Archer (2000) introduces a stratified view of 'the subject' whose different properties and powers (Personal Emergent Power) emerge at each level: the self, the person, the agent and the actor. Among these, the self - the continuous sense of being one and the same subject - emerges early in life and is the source of reflexive selfconsciousness that lasts throughout life. The person refers to our unique personal identity. Our 'social selves' - the agent and the actor - emerge respectively through our involuntary embroilment in society's role-array and they are dependent upon "the prior emergence of a continuous sense of self and are co-dependent with the emergence of personal identity" (Archer 2000, 255).The process through which agents transform themselves in pursuing social change and obtaining a social identity is summarised in Figure 1.

Primary Agents are defined as "collectivities sharing the same life-chances" (Archer 2000, 263) and therefore everyone is necessarily a Primary Agent by being assigned to positions on society's distributions of scarce resources (Archer 2000). The pre-existence of those structures, which shape the situation in which we find ourselves, impinges upon us without our compliance, consent or complicity, leading to the aggregate status of Primary Agents. In order to reshape or retain the structural and/or cultural features, collective action that has systemic consequences has to be developed. 'Corporate Agents' are those "who articulate shared interests, organise for collective action, generate social movements and exercise corporate influence in decision-making" (Archer 2000, 266). Archer distinguishes Primary Agents from Corporate Agents as Primary Agents neither express interests nor organise for their strategic pursuit, either in society or in a given institutional sector. However, for Primary Agents, Archer emphasises that "to lack of a say in systematic organisation and re-organisation is not the same as have no effect upon it, but the effects are unarticulated in both senses of the word - un-coordinated in action and unstated in aim" (Archer 2000, 266). Therefore, Primary Agents are not deemed intrinsically passive but their passivity represents a suspension of immediate action, often a deliberate suspension (Archer 2000, 266). By contrast, Corporate Agents are active in the sense that they are attempting to bring about certain outcomes, rather than being objects to whom things happen.

The social and structural transformations depicted in this process are not the focus in this study given the scale and time involved for these to emerge in practice. However, the process of socialisation of Primary Agency, in particular from Quadrants 2 and 3 in Figure 1, can be employed to analyse the data in order to reach 


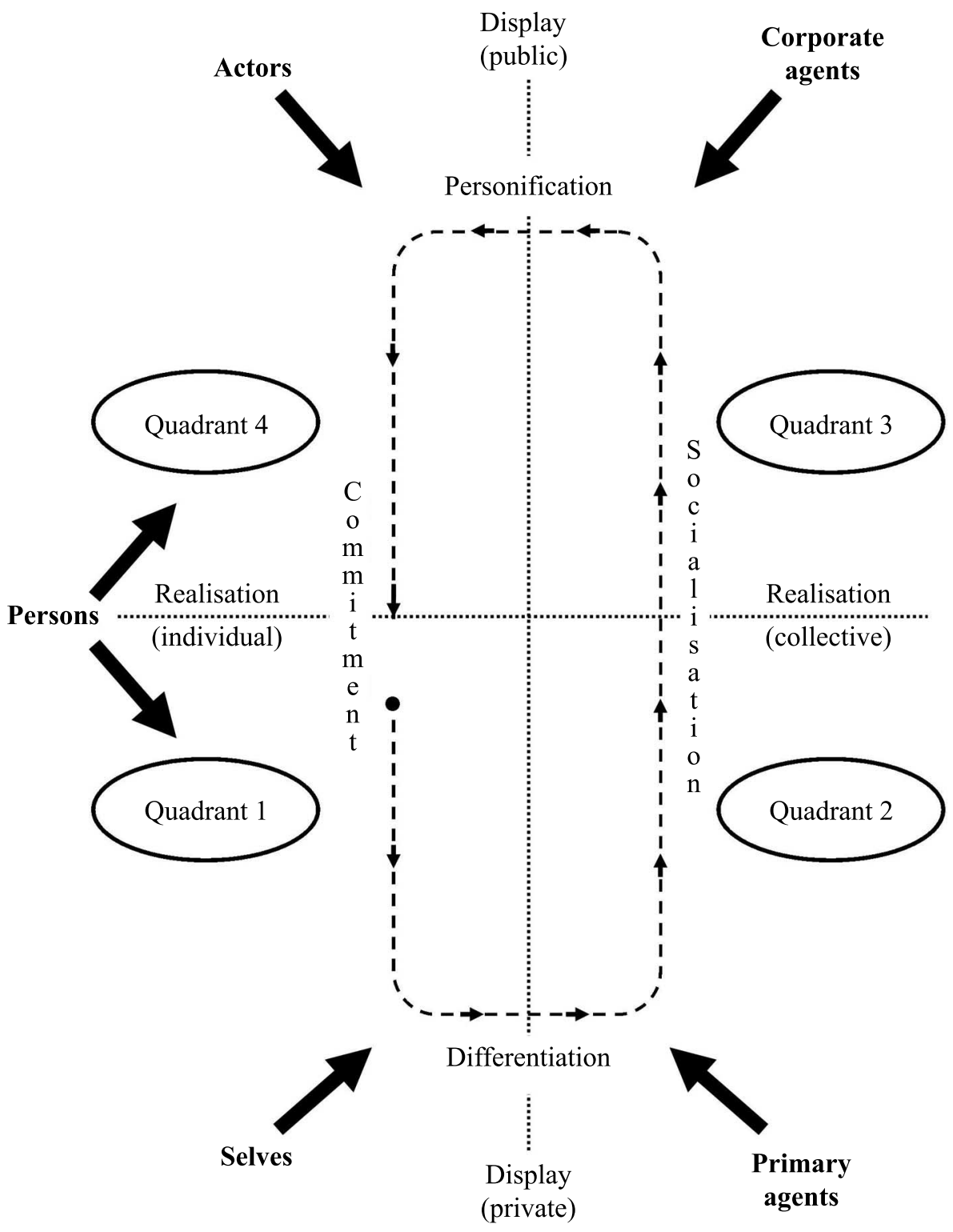

Figure 1. Realism's account of the development of the stratified human being. Source: Archer (2000, 260).

an understanding of the emergence of an e-learning community as revealed in this study.

In Figure 2, the upper part of the figure depicts Archer's model of the acquisition of social identity as represented in Figure 1, showing the process of socialisation of Primary Agents undergoing transformation into Corporate Agents. Corresponding to that, the lower part shows the formation of a learning community in an e-learning context. 


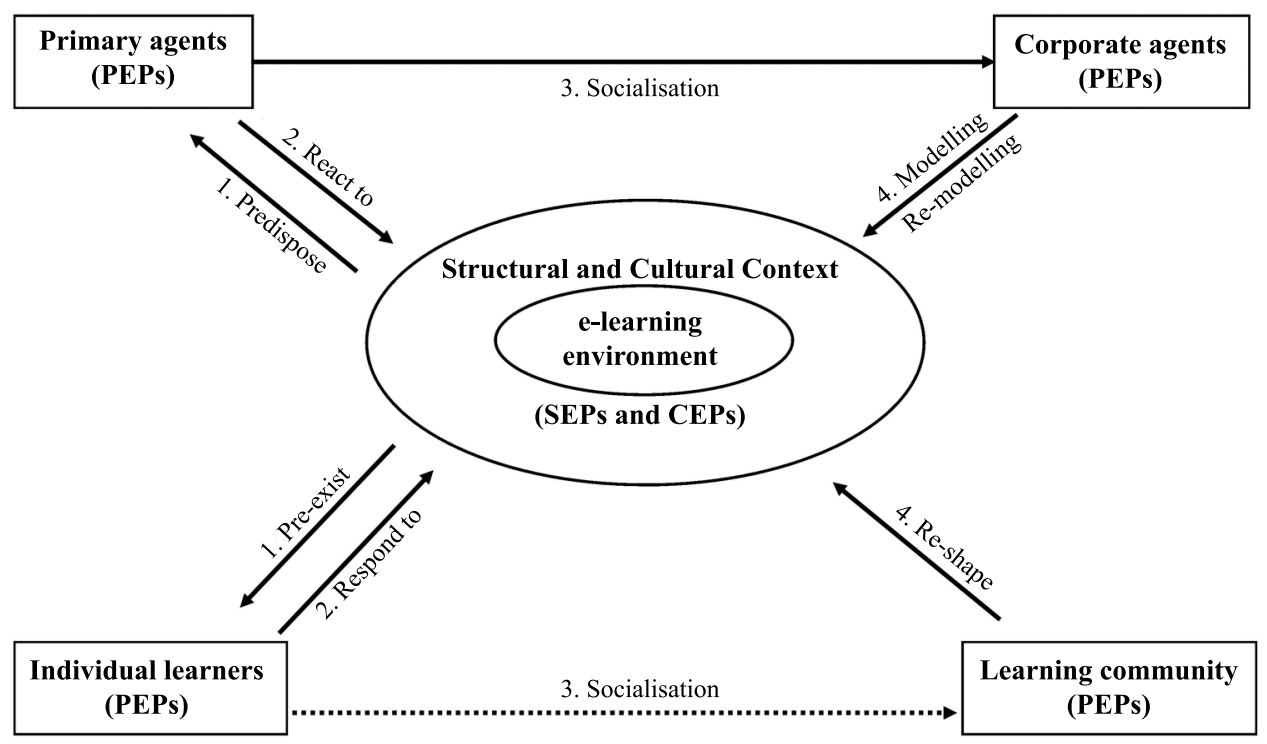

Figure 2. The emergence of the (e-)learning community.

Note: CEP, Cultural Emergent Power; PEP, Personal Emergent Power; SEP, Structural Emergent Power.

In an e-learning environment, learners come to study on an online course as individuals. The pre-existence of course content, structure, pedagogy, teaching method, communicative means and technology adopted, plus the institutional environment and culture, have shaped the course context in which e-learners are going to learn. Individual learners, in this sense, can be seen in this context as Primary Agents, who are assigned to similar positions in the course and share similar resources available. However, because of learners' personal power - reflexivity - they act back on these structural settings. In forming a learning community, individual learners may (or may not) undergo 're-grouping' at some precise point by articulating their shared interests or organising collective actions with others to achieve common aims.

Wenger (1998) characterises three dimensions of a community of practice: mutual engagement, a joint enterprise and a shared repertoire. Common features can be found between these characteristics and Archer's theorisation. By organising their strategic pursuit, Corporate Agents obtain their membership in a community of practice. This mutual engagement does not require homogeneity but does require being included in what matters (Wenger 1998). The things that matter are referred to as a joint enterprise, which in turn can create a source of community coherence; and for Archer (2000) the thing that matters is an articulated shared interest. Both authors believe that this joint enterprise results from a negotiated response to the situations in which people find themselves. Moreover, they both recognise the pre-existence of structural and cultural factors (Structural Emergent Powers and Cultural Emergent Powers ) in shaping the situations, plus specific resources being available and the existence of constraints. Archer (2000), in particular, specifies that the distribution of those available resources can include both the material and the ideational. The power to form collective action (Archer 2000) or achieve mutual engagement towards a joint enterprise (Wenger 1998) resides with the participants. For Archer, the nature of our human 
powers - reflexivity - means that "the actual outcomes are matters of secondary determination, governed by our inner deliberations about such obstructions and facilitations, under our own descriptions. We often have the capacity to suspend both" (Archer 2007, 8). In a similar vein, Wenger argues that:

Yet even when the practice of a community is profoundly shaped by conditions outside the control of its members, as it always is in some respects, its day-to-day reality is nevertheless produced by participants within the resources and constraints of their situations. It is their response to their conditions, and therefore their enterprise. $(1998,79)$

Attention has thus been drawn to this "indigenous enterprise of community of practice" (Wenger 1998, 79) or "human power in mediating structural factors" (Archer 2000, 8) without ignoring the influence of structural and cultural factors (Structural Emergent Powers and Cultural Emergent Powers ). Therefore, examining the interplay of these two set of powers in the e-learning contexts, with the aid of this diagram, can assist with interpreting and understanding the findings of the study regarding the emergence of a learning community in the two cases, as is unfolded in the following discussion.

\section{The emergence of an e-learning community}

According to the discussion above, a learning community emerges when a group of individual learners has articulated its shared interest or joint enterprise through negotiation, and organised strategic pursuit to achieve this stated target, and its collective actions in turn have exerted effects to bring about situational change.

There was no lack of common interest in the course among the learners in Case One, such as interest in the subject knowledge and improving their own working efficacy. Moreover, learners were also evidently eager to share their learning and working experience, exchange ideas and solve problems together. The face-to-face informal discussions during the course study with their peers in the same school were very intensive. As this participant indicated in a focus group interview:

I found the discussions with colleagues in the school who are also in the programme have been much more helpful, rather than trying to find someone you don't know to talk to from the [online] forum, even these colleagues might be taking different modules. (Case One: Students Focus Group 4)

Unfortunately, the number of participants taking the same module in the same school was small (less than five on average). Therefore, some problems can be highlighted, such as the poorly functioning communicative tools promoted by the course designers, and too limited a number of participants taking the same module so that these common interests failed to be articulated and developed into a workable joint enterprise. Consequently, actions remained un-coordinated and individual learners did not progress beyond being Primary Agents.

In contrast, in Case Two, despite the more content-focused course design, the negotiation and achievement of the joint enterprise was enabled through intensive networking with the aid of different communicative means both through and outside the course venue, giving rise to coherence of practice in a learning community. Extensive activities, including information sharing and exchanging, dealing with assignments and examinations and solving problems, were in evidence in the online forums and group space. Geographical proximity also helped group members interact with 
each other more directly. Some of them set up face-to-face meetings and study groups with peers in the same city. Going beyond the face-to-face meetings and discussions of the course, the class committee also organised some social events and activities from time to time to gather people together. One of the most enjoyable and positive experiences reported by many learners in this case was meeting their peer students and developing relationships with them. Wenger (1998) has argued that what it takes for a community of practice to cohere enough to function can be very subtle and delicate, and this coherence also requires work. In this case, the course provided a platform, although unintentionally, where learners were able to link up with each other, and share their common interests and concerns as a means of mutual support to help them successfully navigate the course.

Archer (2000) argues that the influence of structural factors is mediated by the human power of reflexivity. This can explain the gap found between the course designers' intentions and learners' actual experiences in both cases. Similarly, Wenger (1998) argues that an enterprise can never be fully determined by an outside mandate, by a prescription, or by any individual participant, but can only be negotiated by the community that conditions and resources practice, and whose demands shape that practice. It is 'their' (learners') learning community to emerge where 'their' (learners') negotiated joint enterprise becomes necessary as a source of community of coherence.

On the other hand, individual agents cannot make whatever they want from the situation but are constrained by the ideational and material resources available (Archer 2000). Wenger (1998) has argued that whatever it takes to make mutual engagement possible is an essential component of any practice. The limited number of students taking the same module made it difficult for learners in Case One to find peers to 'share information' with. Consequently, the process of negotiation and discussion was inhibited, particularly since this limitation was combined with the short duration of the programme, weakly defined learning objectives and assessment methods, unfamiliarity with the pedagogy and communicative tools employed, as well as language barriers. What we observe here, therefore, is an example of agential power constrained by a combination of structural powers. To engage with these constraints and attempt to overcome them would have required a greater commitment to the programme than the students felt was justified when balancing its potential professional value against other demands in their daily lives (cf. Archer 2000, 297). The significance of user numbers in increasing engagement as identified in this study is in line with Crook's (2008) observation that the growth in the sheer number of Internet users contributes to the popularity of social networking where people share and exchange information and interact with each other.

Furthermore, learners who gained their membership in the learning community were apparently those who had transformed from being Primary Agents to Corporate Agents: "who know what they want, can articulate it to themselves and to others, and have organised in order to obtain it, can engage in concerted action to reshape or retain the structural and/or cultural features in question" (Archer 2000, 265). The learners' experiences in Case Two have illustrated such agential power in articulating shared interests, organising for collective actions and exercising corporate influence in decision-making, such as their collaborative efforts and success in getting the poorly delivered lectures re-organised. Learners in this case have thus been strategically involved in the modelling or re-modelling of the course structure and culture by making themselves heard in the decision-making arena. 
Structural constraints and enablements in the emergence of a learning community also manifested themselves in a more subtle way. Research conducted in conventional classroom settings with Chinese learners, such as that of Lee (1999) and Zhou, Knnoke, and Sakamoto (2005), suggests that acquaintance and personal familiarity with peer students influence students' interactions with each other. This study shows that this applies equally to an e-learning environment. The development of warm and trusting interpersonal relationships among learners evidently helped the emergence of the learning community to a great extent. As one interviewed teacher expressed it: "I found they actually know each other very well, even the personal life and family issues". This familiarity with their peer students might have been gained from their informal chatting using the online instant messenger, social activities organised by the class committee, and so forth: 'Sharing meaning' has become motivation for learners to collaborate, as Crook (2002) similarly found in his study. Learners share information, from the course assignments to the latest gossip. Wenger argues that:

what it takes for a community of practice to cohere enough to function can be very subtle and delicate ... it is difficult to distinguish between the value of a specific piece of information and the value of the atmosphere of friendliness they create, or between bits of talk about work and the personal exchanges that are woven into their conversations. $(1998,74)$

The social aspects of interaction are thus crucial to building up trusting relationships, and knowledge of other participants is a central part of these aspects. However, I argue that the preference for communicating with people whom they know should not be seen as a feature exclusive to Chinese learners, as some, such as Bond (1991), have assumed. Research has already found that in a computer-conferencing setting, people might be put off by the impression of a large lurking, anonymous audience, who could read their contributions (Grint 1989). Similarly, Wegerif (1998) found in his study that the social dimension is important to the effectiveness of asynchronous learning networks and needs to be taken into account in the design of courses.

To understand this from an Archerian analysis of learners' reflexivity it was also necessary to draw on key concepts within Chinese 'traditional' philosophies and experiences of learning and social engagement. As discussed previously, the central concept in Confucian philosophy, 'Ren' (仁, benevolence), represents balanced and harmonised human relationships, and trust is the critical ingredient of all dependent social interactions in order to achieve this balanced and harmonised human relationship. 'Ren' (仁) is seen as being manifested in Chinese educational tradition and practice in many different aspects: learning is a cooperative process between teacher and student; there is a strong sense of trust and responsibility within the teacher-student relationship; students' spontaneous collaborations outside the classroom, small group work involving collaboration and cooperation seems to be a 'natural' way to structure learning among ethnic Chinese learners (Li 2009). Unfortunately, a warm and close relationship between teacher and students failed to be achieved in both cases in this study. In Case One, it was found more difficult to achieve such trusting interpersonal interaction through computer-conferencing communication. One-to-one communications with the tutors were sought by the learners but discouraged by tutors. In Case Two, the one-way teaching process left learners' desire to know and interact with their teacher unfulfilled. The online learning environment made it difficult for learners to make any informal contacts with teachers as they might have been used to doing in their previous learning experience. 
Moreover, the findings suggest that developing a trusting human relationship also entails mutual understanding, which was not achieved well between teachers and learners in both cases. The origins of the gap were found to be in the diverse understandings held by the course designers, teachers and learners about learners' needs, and their preferences with regard to learning strategy and technology usage. In particular, in Case One, rather than examining the structural constraints in the course design and other factors in the e-learning settings, learners were harshly judged to "lack autonomy" because "the traditional ways of Chinese pedagogic approaches ... are typically teacher-driven and teacher-centred" (Case One: eTutor Report I). This stereotyping of the learners and misunderstanding of their learning behaviours inhibited the giving of truly valuable support, thus impeding the building of a trusting relationship between tutors and learners in the e-learning environment. In Case Two, the limited interaction between teacher and learners also prevented such mutual understanding from developing. By contrast, the most enjoyable experiences reported in both cases were the warm and trusting relationships developed with peer learners, which in Case Two formed the basis of a learning community.

\section{Conclusions}

The forming of a learning community is a socialisation process for a group of individual learners, during which they express their interests and organise the strategic pursuit of their common target. To facilitate such a socialisation process, there should be resources available, both ideational and material, in the e-learning settings. For instance, an e-learning course can be used as a platform where learners can engage with those who have similar interests to themselves. As has been found in classroom settings, spontaneous collaboration and small group work involving collaboration and cooperation seemed to a natural way to structure learning among ethnic Chinese learners. At least, it is seen as inappropriate to attribute an unsatisfactory implementation of a collaborative course design as due to a default 'learners' predisposition'. When there are teaching innovations that are fresh to learners, the right question to ask might be what kind of extra support should be given to them. Moreover, the development of trusting human relationships is a key to the emergence of the learning community because relationships are identified in this study as a driving force in the learning process. Technologies expand the availability of such relationships across the traditional boundaries of time and space. At the same time, they set up barriers to be overcome by removing more direct human contacts during the interactions. Therefore, bringing moral support into the e-learning environment should be seen as a crucial issue.

The findings in this study have also indicated the significance of human relationships in an e-learning environment influencing learners' learning experiences; in particular, in forming a learning community. The strong sense of responsibility and personal care marked in teacher-student relationships in Chinese cultural and educational tradition is difficult to achieve in an e-learning environment where the opportunities for informal social interaction are reduced. Any change in the balance of power in relationships between teacher and learner does not come automatically as a result of adopting a new pedagogical approach in e-learning design but depends on trusting human relationships being established. This concept of 'Ren' in Chinese philosophical tradition thus has significant meaning in an e-learning environment. The building up of 'Ren' (仁) in e-learning settings calls for effective 
interpersonal interactions, with the aid of communication technologies. 'Social presence' in communication is perceived as important for shortening the interpersonal distance and achieving mutual understanding. Therefore, human contact cannot, and should not, be replaced by contact with or through a machine. Moreover, a trusting relationship can only be achieved in the absence of prejudice and stereotyping but with an understanding of the possible different preferences in learning approaches between different cultures and social contexts.

\section{References}

Archer, M. 1996. Culture and agency: The place of culture in social theory. Cambridge: Cambridge University Press.

Archer, M. 2000. Being human: The problem of human agency. Cambridge: Cambridge University Press.

Archer, M. 2007. Making our way through the world: Human reflexivity and social mobility. New York: Cambridge University Press.

Bond, M.H. 1991. Beyond the Chinese face: Insights from psychology. Hong Kong: Oxford University Press.

Cole, M., and Y. Engeström. 1993. A cultural-historical approach to distributed cognition. In Distributed cognitions: Psychological and educational considerations, ed. G. Saloman, 1-46. Cambridge: Cambridge University Press.

Conole, G. 2007. An international comparison of the relationship between policy and practice in e-learning. In Handbook of e-learning research, ed. R. Andrews and C. Haythornthwaite, 286-310. London: Sage.

Conole, G., J. Smith, and S. White. 2007. A critique of the impact of policy and funding. In Contemporary perspectives in e-learning research: Themes, methods and impacts on practice, ed. G. Conole and M. Oliver, 38-54. London: Routledge.

Conole, G., M. De Laat, T. Dillon, and J. Darby. 2006. JISC LXP: Student experiences of technologies. Final report of JISC-funded LXP Project. Southampton: University of Southampton. http://www.jisc.ac.uk/elp_learneroutcomes.html.

Creanor, L., D. Gowan, C. Howells, and K. Trinder. 2006. LEX: The learner experience of elearning - Final project report. Glasgow: Glasgow Caledonian University. http:// www.jisc.ac.uk/uploaded_documents/LEX\%20final\%20Report_August06.pdf.

Crook, C. 2002. The campus experience of networked learning. In Networked learning: Perspectives and issues, ed. C. Steeples and C. Jones, 293-308. London: Springer-Verlag.

Crook, C. 2008. What are web 2.0 technologies, and why do they matter? In Education 2.0? Designing the web for teaching and learning: A commentary by the Technology Enhanced Learning phase of the Teaching and Learning Research Programme (TLRPTEL), ed. N. Selwyn, 6-9. http://www.tlrp.org/pub/commentaries.html.

Grint, K. 1989. Accounting for failure: Participation and non-participation in CMC. In Mindweave: Communication, computers and distance education, ed. R. Mason and A. Kaye, 182-92. Oxford: Pergamon Press.

Lave, J., and E. Wenger. 1991. Situated learning: Legitimate peripheral participation. Cambridge: Cambridge University Press.

Lee, C. 1999. Learning through tutorial discussion and learners' culture: Some preliminary observations from the views of Hong Kong Chinese Tertiary students. Language, Culture and Curriculum 12, no. 3: 255-64.

Li, Z. 2009. Making our way through the e-world: Chinese adult e-learners' learning experiences. PhD diss., University of Southampton, UK.

Timms, S., R. O’Leary, E. Weedon, and K. Martin. 2004. A multi-disciplinary, holistic approach to networked learning research: A critique of a large-scale empirical study into student online learning experiences. Paper presented at Networked Learning Conference, April 5-7, in Lancaster University, UK.

Wegerif, R. 1998. The social dimension of asynchronous learning networks. Journal of Asynchronous Learning Networks 2, no. 1. http://www.aln.org/alnweb/journal/vol2_issue1/ Wegerif.pdf. 
Wenger, E. 1998. Communities of practice: Learning, meaning and identity. Cambridge: Cambridge University Press.

Zhou, Y.R., D. Knoke, and I. Sakamoto. 2005. Rethinking silence in the classroom: Chinese students' experiences of sharing indigenous knowledge. International Journal of Inclusive Education 9, no. 3: 287-311. 